La Revue

des Droits

de l'Homme

\section{La Revue des droits de l'homme}

Revue du Centre de recherches et d'études sur les droits fondamentaux

Actualités Droits-Libertés | 2014

\title{
Quand la liberté d'établissement fait la courte échelle au regroupement familial
}

Droit au regroupement familial (Union européenne)

Anne Soisson et Pascal Schumacher

\section{(2) OpenEdition}

Édition électronique

URL : http://journals.openedition.org/revdh/853

DOI : $10.4000 /$ revdh.853

ISSN : 2264-119X

Éditeur

Centre de recherches et d'études sur les droits fondamentaux

Référence électronique

Anne Soisson et Pascal Schumacher, "Quand la liberté d'établissement fait la courte échelle au regroupement familial », La Revue des droits de l'homme [En ligne], Actualités Droits-Libertés, mis en ligne le 22 juillet 2014, consulté le 19 avril 2019. URL : http://journals.openedition.org/revdh/853 ; DOI : 10.4000/revdh.853

Ce document a été généré automatiquement le 19 avril 2019

Tous droits réservés 


\title{
Quand la liberté d'établissement fait la courte échelle au regroupement familial
}

Droit au regroupement familial (Union européenne)

\author{
Anne Soisson et Pascal Schumacher
}

1 Le regroupement familial s'inscrit dans le cadre plus large du droit au respect de la vie familiale garanti à l'article 8 de la Convention européenne de sauvegarde des droits de l'homme et des libertés fondamentales ou à l'article 7 de la Charte des droits fondamentaux de l'Union européenne. Longtemps, les juridictions se sont opposées à admettre des motifs de limitation de ce droit, pour ensuite céder à une extension progressive encadrée par l'interprétation stricte des clauses restrictives. Le droit au respect de la vie familiale peut faire l'objet de limitations spécifiques, qui requièrent un ancrage dans les législations nationales et à la condition de ne pas porter atteinte à la substance même du droit.

2 Dans les affaires de regroupement familial analysées ci-dessous, on pouvait légitiment s'attendre à ce que l'enjeu réside dans les conditions traditionnelles d'admission de ces restrictions, à savoir - selon le jargon strasbourgeois - le fait notamment d'être « prévue par la loi " et d'être "nécessaire dans une société démocratique». Selon un raisonnement classique, le juge allait d'abord examiner si la restriction était suffisamment accessible et précise pour ensuite mettre en balance les intérêts de l'individu et ceux de la société. Tout au plus, le juge pouvait encore s'attarder sur les obligations positives des autorités étatiques pour déterminer la manière dont l'Etat agit pour permettre au lien familial de se développer. Voilà à quoi pouvait s'attendre le lecteur avisé...

3 L'arrêt Dogan (affaire C-138/13) de la Cour de justice de l'Union européenne (CJUE), traitant d'une affaire de refus de regroupement familial, porte principalement sur la compatibilité entre une exigence linguistique imposée par l'Allemagne depuis 2007 et la clause de «standstill» convenue au début des années 1970 dans le cadre de l'accord d'association avec la Turquie et interdisant l'introduction de nouvelles restrictions à la liberté d'établissement. 
Dans le cas d'espèce, la requérante, Mme Naime Dogan, ressortissante turque résidant en Turquie, souhaite rejoindre son époux en Allemagne. Celui-ci y vit depuis 1998 et disposait, depuis 2002, d'un titre de séjour à durée déterminée, remplacé ensuite par un titre de séjour à durée indéterminée. $\mathrm{M}$. Dogan dirige en Allemagne une société à responsabilité limitée dont il est l'actionnaire majoritaire.

5 A plusieurs reprises, Mme Dogan a introduit des demandes à l'ambassade d'Allemagne à Ankara afin d'obtenir un visa au titre de regroupement familial. A chacune de ses demandes, elle joignait des attestations de connaissances élémentaires de langue allemande, attestations exigées par la loi allemande relative au séjour, au travail et à l'intégration des étrangers sur son territoire fédéral. Même si, objectivement, elle satisfaisait aux exigences linguistiques, les autorités allemandes lui ont refusé à plusieurs reprises la délivrance d'un visa. Elles ont fait valoir que Mme Dogan était analphabète et que, par conséquent, ses réussites aux examens linguistiques étaient le fruit du hasard. Face à ces refus constants, la requérante a introduit un recours devant le tribunal administratif de Berlin.

Le Verwaltungsgericht de Berlin a décidé de surseoir à statuer et posé deux questions préjudicielles à la CJUE. Dans un premier temps, il a demandé à la Cour si une mesure nationale, introduite après l'entrée en vigueur de la clause de «standstill » et qui exige du conjoint d'un ressortissant turc résidant en Turquie de prouver l'acquisition de connaissances linguistiques élémentaires de la langue allemande avant d'entrer sur le territoire allemand au titre de regroupement familial, s'oppose à l'article 41 de la clause de «standstill». Dans un deuxième temps, il a cherché à savoir si pareille mesure allemande se justifie aux termes de l'article 7(2) de la directive 2003/86 sur le droit au regroupement familial. Cet article permet aux Etats membres d'exiger des ressortissants de pays tiers que ces derniers se conforment aux mesures d'intégration afin de bénéficier du droit au regroupement familial.

7 Dans un jugement relativement court, la CJUE affirme qu'une telle exigence linguistique s'oppose à l'article 41 de la clause de «standstill» en constituant une restriction additionnelle à l'exercice de la liberté d'établissement par les ressortissants turcs. En effet, comparées aux règles applicables lors de l'entrée en vigueur de la clause de «standstill», les exigences linguistiques rendent le regroupement familial plus difficile en durcissant les conditions de première admission du conjoint d'un ressortissant turc sur le territoire de l'Etat membre concerné.

8 La première partie de cette analyse se concentrera sur l'exercice de la liberté d'établissement des ressortissants turcs $\left(1^{\circ}\right)$, alors que la deuxième partie approchera la question du régime des restrictions à cette liberté fondamentale $\left(2^{\circ}\right)$.

\section{1\%- L'exercice de la liberté d'établissement des ressortissants turcs : Une condition de base à l'interdiction de nouvelles mesures rendant le regroupement familial plus difficile}

9 Cette affaire s'inscrit dans le cadre des négociations d'une éventuelle adhésion de la Turquie à ce qui était, dans les années soixante encore, la Communauté économique européenne. Pendant cette période, l'accord d'Ankara de 1963 et son Protocole 
additionnel de 1970 ont été signés. Alors que le premier vise uniquement à renforcer les relations économiques et commerciales entre la Turquie et la CEE et ne donne pas aux citoyens turcs un droit matériel d'entrer ou de résider dans un Etat membre de l'Union ${ }^{1}$, le second permet aux travailleurs indépendants et prestataires de services turcs de bénéficier d'une clause de «standstill» interdisant aux Etats membres d'imposer des exigences financières ou procédurales plus strictes que celles qui étaient déjà en vigueur lors de la conclusion de l'accord. Par ailleurs, en vertu de la décision 1/80 du Conseil d'association établi par l'accord d'Ankara, les membres de la famille de ressortissants turcs ont droit à un traitement privilégié, notamment en matière de regroupement familial.

10 Sur cet arrière-fond, la CJUE a répondu à la première question préjudicielle en suivant un raisonnement en trois étapes donnant forme à la clause de «standstill». Rappelant sa jurisprudence constante ${ }^{2}$, elle met tout d'abord en avant que cette clause interdit l'introduction de toute nouvelle mesure ayant pour objet ou pour effet de soumettre l'exercice par un ressortissant turc de la liberté d'établissement ou de la libre prestation de services à des conditions plus restrictives que celles applicables lors de l'entrée en vigueur de cette clause. Par la suite, en se basant sur une jurisprudence déjà établie ${ }^{3}$, elle précise que cette interdiction s'étend également à des mesures portant sur les conditions de fond et/ou de procédures applicables aux ressortissants turcs exerçant la liberté d'établissement ou étant prestataires de service en matière de première admission sur le territoire de l'Etat membre en question. En dernier lieu, elle met en évidence que la clause de «standstill » ne peut être invoquée que lorsqu'elle est le corollaire de l'exercice d'une activité économique.

11 Ayant établi ce cadre, la Cour l'applique au cas d'espèce et conclut que, eu égard à l'exercice de la liberté d'établissement par M. Dogan, l'exigence linguistique à laquelle est soumise Mme Dogan est non conforme avec la clause de «standstill». Selon la Cour, cette mesure, introduite après l'entrée en vigueur du protocole, constitue un durcissement des conditions d'admission en matière de regroupement familial qui mène à une situation dans laquelle M. Dogan doit choisir entre son activité professionnelle en Allemagne et une vie de famille en Turquie.

Bien que, parmi ces 11 premiers paragraphes, les deux derniers (point 34 et 35) frôlent prudemment le droit au respect de la vie familiale, il ne peut pas être déduit de cet arrêt un renforcement général du droit au regroupement familial et encore moins du droit au respect de la vie familiale des ressortissants tiers. En effet, la base de l'argumentaire de la CJUE se fonde irrémédiablement sur le seul exercice de la liberté d'établissement. Elle rappelle à chacune des étapes mentionnées ci-dessus que les difficultés liées au regroupement familial par l'exigence de connaissances élémentaires de la langue allemande est considérée comme une nouvelle restriction parce qu'elle risque de menacer la liberté d'établissement exercée par M. Dogan. La Cour ne détaille pas les conséquences sur la vie familiale d'un ressortissant de pays tiers au regard des mesures prises pour la délivrance de visas. Elle ne fait pas non plus allusion, ni à l'article 8 de la Convention européenne de sauvegarde des droits de l'homme et des libertés fondamentales, ni à l'article 7 de la Charte des droits fondamentaux de l'Union européenne. Il ressort d'une interprétation a contrario qu'un membre de famille reste soumis à certaines mesures nationales d'intégration en matière de regroupement familial, tant qu'il ne sait pas prouver un lien entre exercice de libertés économiques et maintien de l'intégrité de la vie familiale. 
13 En renforçant les droits au regroupement familial de ressortissants tiers turcs sous l'angle de la liberté d'établissement, la CJUE a suivi l'argumentation proposée par l'avocat général Mengozzi et la Commission, et non pas par les gouvernements allemands et néerlandais ${ }^{4}$. Alors que ces derniers ont été d'avis "qu'une règle telle que celle en cause au principal sur l'exercice de la liberté d'établissement au titre de l'accord d'association est trop éloignée et hypothétique pour pouvoir relever aux fins de l'application de la clause de "standstill » ", l'avocat général a mis en évidence dans sa réponse le lien de principe entre jouissance des libertés fondamentales et intégration familiale, sans référence expresse au droit au respect de la vie familiale.

14 Ayant répondu à la $1^{\text {ière }}$ question, la CJUE s'est démise de son obligation de devoir répondre à la deuxième question et a ainsi évité un examen de compatibilité entre la directive 2003/86 et la subordination de la délivrance d'un visa aux fins du regroupement de conjoints de ressortissants turcs résidant légalement en Allemagne par l'exigence de connaissances élémentaires de l'allemand. Or, comme le démontre l'approche de l'avocat général à cette partie ${ }^{5}$, pareille analyse aurait probablement mené à une réflexion approfondie de la Cour sur le droit au respect de la vie familiale. En effet, le considérant (2) de cette directive met en avant que « les mesures concernant le regroupement familial devraient être adoptées en conformité avec l'obligation de protection de la famille et de respect de la vie familiale qui est consacrée dans de nombreux instruments du droit international. La présente directive respecte les droits fondamentaux et observe les principes qui sont reconnus notamment par l'article 8 de la Convention européenne pour la protection des droits humains et des libertés fondamentales et par la charte des droits fondamentaux de l'Union européenne ».

En outre, il est à noter que l'Allemagne a signé, mais pas encore ratifié, la Charte sociale européenne révisée du Conseil de l'Europe. L'article 19(6) de celle-ci garantit le droit au regroupement familial. Selon le Comité européen des droits sociaux, comité en charge de la mise en œuvre de cette Charte, l'obligation faite aux membres de la famille du travailleur migrant de passer, entre autres, des tests de langues afin d'entrer dans le pays en question ou/et d'y résider constitue une restriction susceptible de priver de son contenu l'obligation posée à l'article 19(6) de la Charte.

L'absence d'un renvoi au droit au respect de la vie familiale est d'autant plus percutant que la CJUE a fait référence, il y a presque deux ans, à l'article 7 de la Charte des droits fondamentaux de l'Union européenne dans un jugement portant sur d'éventuelles limitations au droit au regroupement familial conféré par la Décision $1 / 80 .{ }^{6}$ Alors que, dans l'affaire Dogan, elle ne fait aucune référence au droit au respect de la vie familiale, elle a souligné dans l'affaire Dülger qu'une éventuelle interprétation trop étroite de la Décision $1 / 80$ peut contrevenir au droit au respect de la vie privée et familiale consacrée par l'article 7 de la Charte.

Suite à une coïncidence hors du commun, le même jour que l'affaire Dogan, la Cour européenne des droits de l'homme a rendu plusieurs arrêts ${ }^{7}$ portant sur la procédure de regroupement familial. La Cour martèle que les procédures de regroupement familial sont à mener avec souplesse, célérité et effectivité. Mais, elle précise aussi que la nécessité pour les réfugiés de bénéficier d'une procédure de regroupement familial plus favorable que celle réservée aux autres étrangers fait l'objet d'un consensus à l'échelle internationale et européenne. 

européenne pour introduire des exigences d'intégration pour les "familles non-réfugiées ", consistant par exemple dans des tests linguistiques. Bien que son point d'ancrage traditionnel soit le droit au respect de la vie familiale, la Cour s'est résolue à suivre un raisonnement proche de la CJUE en obligeant les autorités nationales à tenir dûment compte de la situation spécifique des requérants, tant que l'Etat réussit par rapport à l'article 8 CEDH à ménager un juste équilibre entre, d'une part, l'intérêt des requérants et, d'autre part, l'intérêt étatique à contrôler l'immigration.

\section{$2 \%$ - La restriction à la liberté d'établissement des ressortissants turcs : Un encadrement strict des mesures d'intégration comme justification d'une entrave}

Alors que l'avocat général cherche d'abord à esquisser les contours de la définition essentielle de la notion de mesures d'intégration en la distinguant des conditions et des critères d'intégration, la Cour de justice de l'Union européenne précise directement au point 37 de son arrêt les modalités du double conditionnement auxquelles doivent répondre les mesures restrictives à la liberté d'établissement des ressortissants turcs : une raison impérieuse d'intérêt général propre à garantir la réalisation de l'objectif légitime poursuivi qui n'aille pas au-delà de ce qui est nécessaire pour l'atteindre. Il est particulièrement regrettable que la Cour ne se soit pas prononcée sur la distinction avancée par l'avocat général entre, d'une part, des mesures d'intégration en principe admises et, d'autre part, des conditions d'intégration en principe répréhensibles.

Dans le cas d'espèce, le gouvernement allemand a soutenu que les exigences linguistiques demandées aux ressortissants turcs sont justifiées par des raisons impérieuses d'intérêt général, plus particulièrement par la prévention des mariages forcés et la promotion de l'intégration. La CJUE partage un tel raisonnement mais souligne qu' « une disposition nationale telle que celle en cause au principal va au-delà de ce qui est nécessaire pour atteindre l'objectif poursuivi, dans la mesure où le défaut de preuve de l'acquisition de connaissances linguistiques suffisantes entraîne automatiquement le rejet de la demande de regroupement familial, sans tenir compte des circonstances particulières de chaque cas $"^{8}$. Pour être conforme au droit de l'Union, chaque mesure restrictive, en plus de constituer une raison impérieuse d'intérêt général, doit donc être appréciée en fonction de l'ensemble des circonstances pertinentes à chaque cas d'espèce, ce qui en pratique exige une évaluation au cas par cas.

21 C'est donc le test de proportionnalité qui a fait échouer la validation du test linguistique. Il convient de signaler que l'effet de l'arrêt se limite au seul cas soumis à la haute juridiction voire à des tests appliqués dans une logique d'automaticité et de conditionnalité analogues.

En appliquant ces règles au cas de Mme Dogan, il devient évident que les conditions imposées par la loi allemande ne répondent pas aux critères du test avancé par la CJUE. L'avocat général Mengozzi soutient que, compte tenu de son âge, de ses conditions

La Revue des droits de l'homme , Actualités Droits-Libertés | 2014 
économiques et de son milieu social, l'analphabétisme de Mme Dogan constitue un obstacle difficilement surmontable. Par conséquent, la subordination de l'autorisation au regroupement familial de Mme Dogan est disproportionnée par rapport à l'objectif et à l'effet utile poursuivis par la clause de "standstill», mais également par la directive 2003/86'. L'Allemagne ne peut donc plus lui refuser un visa aux fins du regroupement familial.

L'arrêt Dogan n'annonce pas une interdiction généralisée des tests linguistiques menés par les Etats membres à l'égard des ressortissants de pays tiers (ou plutôt turcs), mais il les assortit d'une exigence d'individualisation appelant à un examen de la proportionnalité. Ceci n'empêche pas que son prononcé soit à l'origine de fortes vagues, notamment en Allemagne. ${ }^{10}$ Le ministre allemand concerné a déjà fait savoir que son ministère étudie encore le jugement, mais pourrait éventuellement envisager une extension des restrictions prédéterminées pour des cas extrêmement difficiles. Les discussions qui devront être menées à cet égard au sein de la coalisation au pouvoir seront certainement difficiles. En effet, alors que le parti gauche allemand (SPD) a accueilli favorablement ce jugement et exprimé son espoir de voir la fin de tout examen linguistique préalable au regroupement familial, les partis de droite (CSU et CDU) considèrent toute suppression de tests de langues hostiles à la politique de l'intégration. ${ }^{11}$ Cet arrêt n'est pas la première disposition mettant en place une exception à la justification de connaissances en allemand. Dans sa brochure actuelle sur la preuve d'une connaissance élémentaire de la langue allemande dans le cadre du regroupement des conjoints, le Bundesamt für Migration und Flüchtlinge énumère déjà 9 dérogations distinctes ${ }^{12}$. Certains permis de séjours, certaines nationalités, certaines capacités et certaines situations personnelles font déjà naître un droit à une dérogation. Cette liste risquerait de gagner en ampleur, surtout si la Cour de justice de l'Union européenne et la Cour européenne des droits de l'homme continuent sur leurs avancées jurisprudentielles.

Toutefois, les arrêts mentionnés ne permettent pas de verser dans l'euphorie. Les juges semblent hésiter pour renforcer les exigences du droit au respect de la vie familiale dans le cadre d'un regroupement familial. A la Cour de Luxembourg, ce droit fondamental n'est pas le premier angle d'attaque pour assoir son jugement. On peut certainement argumenter que le tribunal de Berlin ne lui a pas posé cette question, ce qui à son tour appelle la réplique selon laquelle la Cour ne s'est pas toujours imposé pareille retenue dans le passé.

A la rigueur, pourrait-on insinuer que ce droit ne doive pas nécessairement se matérialiser sur le territoire de l'Union européenne, mais qu'il pourrait se concrétiser dans le pays tiers tant qu'on n'est pas dans un scénario de protection internationale ? Dans le droit de l'Union, les libertés fondamentales de nature économique semblent, en quelque sorte, l'emporter sur ce droit au respect de la vie familiale, car une restriction injustifiée à leur encontre va donner droit au regroupement familial. En définitive, dans l'arrêt Dogan, la CJUE ne s'est pas avancée sur le terrain d'une prohibition absolue des tests linguistiques, elle s'est limitée à entériner une exigence d'individualisation. En outre, un certain danger semble guetter dans le sillage des jurisprudences 
supranationales, puisqu'on note une certaine fragmentation en fonction des catégories de migrants, risquant d'aboutir à une typologie de migrants jouissant tous de droits et de libertés différents.

Finalement, la notion d'intégration ne constitue pas une notion communautaire, car la politique d'intégration relève de la compétence des Etats membres qui, eux, se réfèrent à des critères antérieurs, préexistants à la date de l'entrée en vigueur de la directive 2003/86, ce qui à l'avenir ne va pas faciliter la tâche du juge luxembourgeois. L'unique terrain d'entente entre Etats membres sur la question linguistique s'est concrétisé par l'adoption des principes de base communs en matière de politique d'intégration des migrants dans l'UE par le Conseil Justice et Affaires intérieures de novembre 2004 et qui constituent le fondement des initiatives communautaires en matière d'intégration. Le principe de base $\mathrm{n}^{\circ} 4$ se lit comme suit : «Des connaissances de base sur la langue, l'histoire et les institutions de la société d'accueil sont indispensables à l'intégration; permettre aux immigrants d'acquérir ces connaissances est un gage de réussite de leur intégration ».

CJUE, 10 juillet 2014, Dogan, Aff. C-138/13 - Conclusions de l'avocat général Mengozzi

Les Lettres « Actualités Droits-Libertés » (ADL) du CREDOF (pour s'y abonner) sont accessibles sur le site de la Revue des Droits de l'Homme (RevDH) - Contact

\section{NOTES}

1. Affaire C-37/98, Savas, 11 mai 2000 ; CJCE, affaire C-16/05, Veli Tum et Mehmet Dari, 20 septembre 2007 ; CJUE, affaire C-186/10, Oguz c. Secretary of State for the Home Department, 21 juillet 2011.

2. Affaire C-186/10, Dereci, 15 novembre 2011, CJCE, point 88 - Lire ADL du 3 décembre 2011

3. Affaire C-186/10, Oguz, 21 juillet 2011, CJCE, point 55.

4. Affaire C-138/13, Dogan, 30 avril 2014, Conclusions de l'avocat général Mengozzi.

5. Ibid., point 47.

6. Affaire C-451/11, Dülger, 19 juillet 2012, CJCE, point 53.

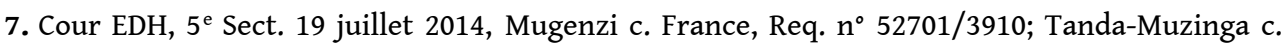
France, Req. $n^{\circ}$ 2260/10, Senior Longue et autres c. France, Req. $n^{\circ}$ 19113/09.

8. Affaire C-138/13, Dogan, 10 juillet 2014, CJUE, point 38.

9. Affaire C-138/13, Dogan, 30 avril 2014, Conclusions de l'avocat général Mengozzi, point 60.

10. Lire Annette Meiritz, «Streit um Zuwanderung: Deutschtest-Zwang spaltet die Koalition », in Der Spiegel, 14 juillet 2014. 
11. Ibid.

12. http://www.bamf.de/SharedDocs/Anlagen/DE/Publikationen/Flyer/ehegattennachzugfr.pdf

\section{RÉSUMÉS}

En ce mois de juillet, en l'espace de seulement quelques jours, plusieurs arrêts ont été rendus, tant à Luxembourg qu'à Strasbourg, qui prima facies semblent renforcer la portée du droit au regroupement familial. Si les verdicts des juridictions supranationales sont favorables aux requérants, un examen minutieux des arrêts laisse craindre une victoire à la Pyrrhus. En effet, plusieurs passages tempèrent la portée protectrice de l'approche des juges et certains éléments viennent même préciser les limites du droit au regroupement familial. Au fil de la lecture, l'euphorie cède la place à un réalisme pragmatique dont l'aboutissement amène à s'interroger sur la nature et la place du droit fondamental au respect de la vie familiale.

\section{AUTEURS}

\section{ANNE SOISSON}

Attachée " Immigration » à la Représentation permanente du Luxembourg auprès de l'Union européenne (NB : Les réflexions contenues dans cet article sont imputables à leurs auteurs et n'engagent pas d'autres organismes ou personnes)

\section{PASCAL SCHUMACHER}

Conseiller « Justice et affaires intérieures » à la Représentation permanente du Luxembourg auprès de l'Union européenne (NB : Les réflexions contenues dans cet article sont imputables à leurs auteurs et n'engagent pas d'autres organismes ou personnes) 